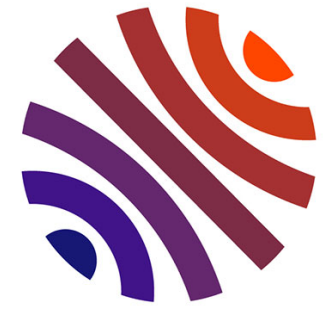 \\ HAL open science
}

\section{Communication: On the origin of the non-Arrhenius behavior in water reorientation dynamics}

\author{
Guillaume Stirnemann, Damien Laage
}

\section{To cite this version:}

Guillaume Stirnemann, Damien Laage. Communication: On the origin of the non-Arrhenius behavior in water reorientation dynamics. Journal of Chemical Physics, 2012, 137 (3), pp.031101. 10.1063/1.4737390 . hal-01339396

\section{HAL Id: hal-01339396 \\ https://hal.sorbonne-universite.fr/hal-01339396}

Submitted on 29 Jun 2016

HAL is a multi-disciplinary open access archive for the deposit and dissemination of scientific research documents, whether they are published or not. The documents may come from teaching and research institutions in France or abroad, or from public or private research centers.
L'archive ouverte pluridisciplinaire HAL, est destinée au dépôt et à la diffusion de documents scientifiques de niveau recherche, publiés ou non, émanant des établissements d'enseignement et de recherche français ou étrangers, des laboratoires publics ou privés. 


\section{AD| $\begin{aligned} & \text { The Journal of } \\ & \text { Chemical Physics }\end{aligned}$}

Communication: On the origin of the non-Arrhenius behavior in water reorientation dynamics

Guillaume Stirnemann and Damien Laage

Citation: The Journal of Chemical Physics 137, 031101 (2012); doi: 10.1063/1.4737390

View online: http://dx.doi.org/10.1063/1.4737390

View Table of Contents: http://scitation.aip.org/content/aip/journal/jcp/137/3?ver=pdfcov

Published by the AIP Publishing

\section{Articles you may be interested in}

Structural dynamics of supercooled water from quasielastic neutron scattering and molecular simulations

J. Chem. Phys. 134, 144508 (2011); 10.1063/1.3578472

Faster proton transfer dynamics of water on $\mathrm{SnO} 2$ compared to $\mathrm{TiO} 2$

J. Chem. Phys. 134, 044706 (2011); 10.1063/1.3509386

A first principles molecular dynamics study of lithium atom solvation in binary liquid mixture of water and ammonia: Structural, electronic, and dynamical properties

J. Chem. Phys. 134, 024519 (2011); 10.1063/1.3511702

Hydrogen transfer and hydration properties of $\mathrm{HnPO} 43-n(n=0-3)$ in water studied by first principles molecular dynamics simulations

J. Chem. Phys. 130, 234502 (2009); 10.1063/1.3143952

Three-dimensional picture of dynamical structure in liquid water

J. Chem. Phys. 112, 1367 (2000); 10.1063/1.480689

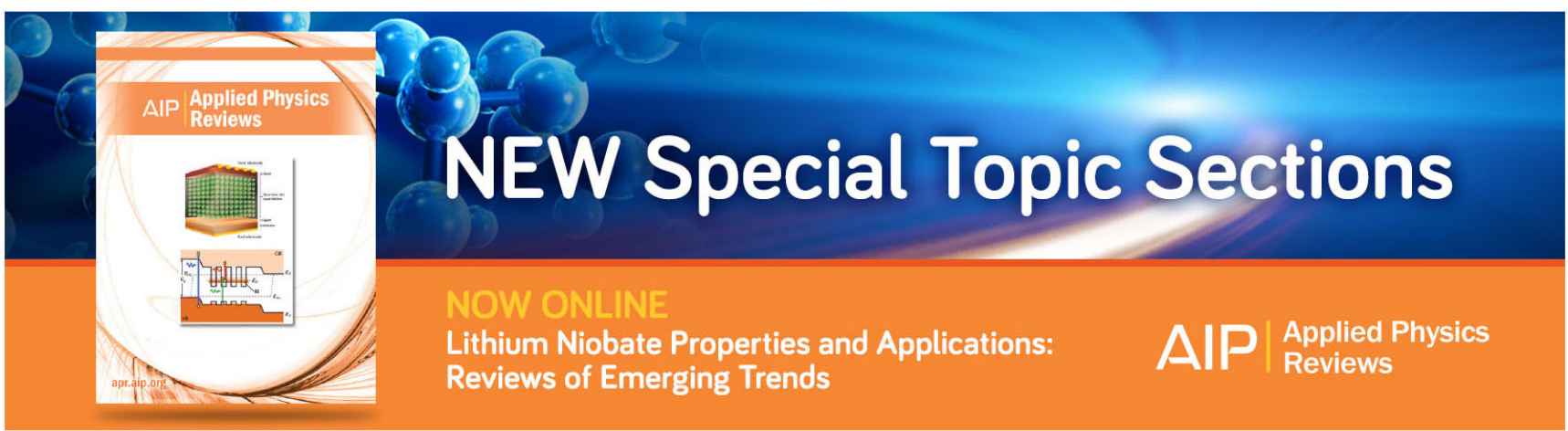




\title{
Communication: On the origin of the non-Arrhenius behavior in water reorientation dynamics
}

\author{
Guillaume Stirnemann ${ }^{\mathrm{a})}$ and Damien Laage ${ }^{\mathrm{b})}$ \\ Chemistry Department, Ecole Normale Supérieure, UMR ENS-CNRS-UPMC 8640, rue Lhomond, \\ 75005 Paris, France
}

(Received 5 June 2012; accepted 2 July 2012; published online 17 July 2012)

\begin{abstract}
We combine molecular dynamics simulations and analytic modeling to determine the origin of the non-Arrhenius temperature dependence of liquid water's reorientation and hydrogen-bond dynamics between $235 \mathrm{~K}$ and $350 \mathrm{~K}$. We present a quantitative model connecting hydrogen-bond exchange dynamics to local structural fluctuations, measured by the asphericity of Voronoi cells associated with each water molecule. For a fixed local structure the regular Arrhenius behavior is recovered, and the global anomalous temperature dependence is demonstrated to essentially result from a continuous shift in the unimodal structure distribution upon cooling. The non-Arrhenius behavior can thus be explained without invoking an equilibrium between distinct structures. In addition, the large width of the homogeneous structural distribution is shown to cause a growing dynamical heterogeneity and a non-exponential relaxation at low temperature. () 2012 American Institute of Physics. [http://dx.doi.org/10.1063/1.4737390]
\end{abstract}

Both the translational and rotational dynamics of liquid water exhibit a non-Arrhenius behavior. ${ }^{1-5}$ The increase of the apparent activation energy $\left(E_{a}\right)$ upon cooling is already perceptible at room temperature and becomes evident in the supercooled liquid. Many explanations have been suggested for this anomalous behavior, including, e.g., a change in the translation and reorientation mechanisms, ${ }^{6}$ the coexistence of high- and low-density liquid (HDL/LDL) structures, ${ }^{7,8}$ the increasingly collective character of water motions at low temperature, ${ }^{9}$ and the freezing of some collective motions. ${ }^{10,11}$ Here, we combine molecular dynamics simulations with an analytic extended jump model (EJM) to study the hydrogen-bond (HB) and reorientation dynamics in liquid water at ambient pressure from high temperatures $(350 \mathrm{~K})$ down to temperatures just above homogeneous nucleation $(235 \mathrm{~K})$.

At each investigated temperature, 40000 water molecules described by the TIP4P/2005 potential ${ }^{12}$ are simulated during up to $5 \mathrm{~ns}$ in the microcanonical ensemble. ${ }^{13}$ Figure 1(a) shows that simulations quantitatively reproduce the non-Arrhenius behavior of the reorientation times measured experimentally. The computed orientation time-correlation functions (tcf) are also in excellent agreement with the available ultrafast measurements, ${ }^{14}$ and increasingly deviate from an exponential decay at low temperature (Fig. 1(b)), which suggests the presence of dynamical heterogeneities. ${ }^{15-17}$ However, in contrast with the popular mode-coupling theory description developed for glass-forming liquids, ${ }^{2}$ at long delays this decay cannot be satisfactorily fit with a stretched exponential (Fig. 1(b)).

For each of our simulations in the $235-350 \mathrm{~K}$ range, the average reorientation mechanism is found to be the same as

\footnotetext{
a) Present address: Department of Chemistry, Columbia University, 3000 Broadway, New York, New York 10027, USA.

${ }^{b)}$ Electronic mail: damien.laage@ens.fr.
}

at room temperature, with a dominant contribution arising from large angular jumps due to $\mathrm{HB}$ exchanges and a minor frame reorientation contribution due to the tumbling of an intact HB. ${ }^{19,20}$ The non-Arrhenius behavior of the reorientation dynamics therefore does not result from a change in the reorientation mechanism at low temperature but instead from the non-Arrhenius kinetics of its two components. Through the entire temperature range, the value of the minor frame contribution is well predicted by the Debye-Stokes-Einstein (DSE) relation and follows the non-Arrhenius behavior of viscosity. ${ }^{13}$ This thus contrasts with the observed breakdown of this relation for the overall reorientation time, ${ }^{21}$ whose dominant jump contribution is non-diffusive, ${ }^{19}$ precluding application of DSE at any temperature. The jump contribution also exhibits a non-Arrhenius behavior, which accounts for most of the increase in the reorientation $E_{a}(\simeq 80 \%$ of $+8 \mathrm{kcal}$ between $350 \mathrm{~K}$ and $235 \mathrm{~K}$, cf. Fig. 1(d)). Since the average jump amplitude is found to be temperature independent, this entirely results from the temperature dependence of the (integrated) jump time $\left\langle\tau_{\text {jump }}\right\rangle$ separating successive jumps. The latter is defined as the relaxation time of the HB survival probability $S(t)$, i.e., the probability not to have jumped to a new HB acceptor after a delay $t,{ }^{13,22}$

$$
\left\langle\tau_{\text {jump }}\right\rangle=\int_{0}^{\infty} S(t) d t .
$$

At low temperature, $S(t)$ displays a non-exponential decay similar to that of the orientation tcf (Fig. 1(c)). ${ }^{13}$ The anomalous reorientation dynamics thus essentially results from the HB jump dynamics, which we now examine.

Jumps between HB acceptors can be fruitfully described as chemical reactions whose kinetics is mostly determined by the free energy costs to elongate the initial $\mathrm{HB}\left(\Delta G_{i}^{\ddagger}\right)$ and for a new HB acceptor to approach and enter in the first hydration shell $\left(\Delta G_{f}^{\ddagger}\right) .{ }^{20}$ Fluctuations in the local water structure may 

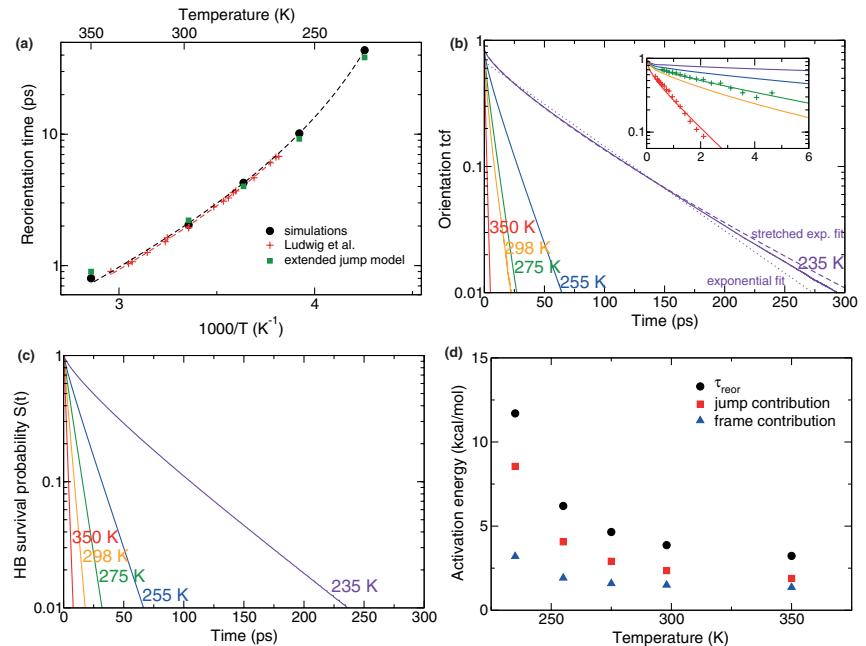

FIG. 1. (a) Arrhenius plot of the integrated water reorientation time from our simulations $\left(\left\langle\tau_{\text {reor }}\right\rangle=\int_{0}^{\infty} d t\left\langle P_{2}[\mathbf{u}(0) \cdot \mathbf{u}(t)]\right\rangle\right.$ where $\mathbf{u}$ is the $\mathrm{OH}$ bond direction and $P_{2}$ is the 2nd Legendre polynomial), from experiments ${ }^{18}$ and from the extended jump model; ${ }^{13,19,20}$ (b) orientation tcf from simulations and experiments (crosses in inset, at $278 \mathrm{~K}$ and $343 \mathrm{~K}$ ) ${ }^{14}$ (c) HB survival probability $S(t)$; and (d) $E_{a}=k_{B} d \ln \left\langle\tau_{\text {reor }}\right\rangle / d(1 / T)$ for $\tau_{\text {reor }}$ and its jump and frame contributions. ${ }^{13}$

alter these free energy barriers and dramatically change the jump time. Many popular parameters developed to describe the water structure were found not to be fully adequate to probe the structural changes affecting the jump dynamics. ${ }^{13}$ These include, e.g., the local density, whose increase facilitates the new partners's approach but hinders the initial bond elongation so that the two effects partly compensate, and the tetrahedral order parameter ${ }^{23}$ which focusses only on the four closest neighbors while the fifth neighbor is critical in jumps.

For the specific case of jump dynamics, ${ }^{13}$ a more pertinent structural description can be provided by the asphericity of the Voronoi polyhedron associated with each water molecule (which includes all points closer to this molecule's oxygen than to any other), defined as $\eta=A^{3} /\left(36 \pi V^{2}\right)$ where $A$ and $V$ are the polyhedron's area and volume. ${ }^{24,25}$ The $g_{\text {OO }}$ radial distribution functions (rdf) in Fig. 2(a) show that molecules with a low- $\eta$ structure experience a weakly structured environment due to the presence of more than four closest neighbors; this both facilitates the initial HB elongation and the approach of a new partner from the second shell, thus accelerating the HB jump. Conversely, high- $\eta$ structures correspond to more tetrahedral (ice-like) arrangements where jumps are slower. The $\eta$ distribution is unimodal and approximately Gaussian at every investigated temperature (Fig. 2(b)). While it slightly shifts to more tetrahedral environments upon
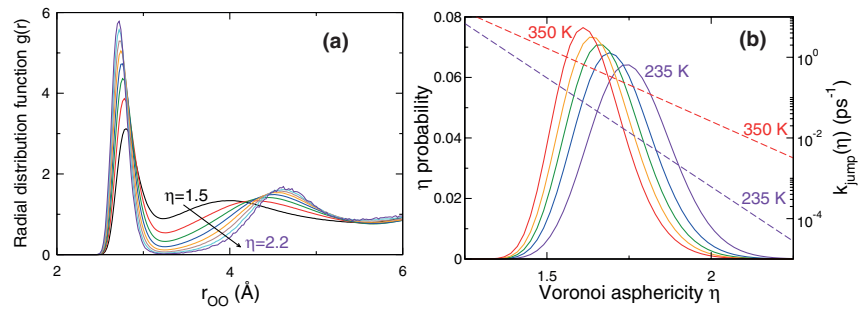

FIG. 2. (a) Radial distribution functions $g_{\mathrm{OO}}(r)$ at $235 \mathrm{~K}$; (b) Voronoi asphericity distributions (solid lines) and $k_{\text {jump }}(\eta, T)$ (dashes, Eq. (5)). cooling, it displays no sign of the two distinct interconverting structures suggested by the LDL/HDL hypothesis ${ }^{7,8}$ (the same behavior was found for the local density ${ }^{13}$ ).

At each temperature and each local structure, the jump free energy barrier can be approximated as ${ }^{22}$

$$
\begin{aligned}
\Delta G^{\ddagger}(\eta, T) & =a+\Delta G_{i}^{\ddagger}(\eta, T)+\Delta G_{f}^{\ddagger}(\eta, T) \\
& \simeq a+k_{B} T \ln \left[\frac{g_{\mathrm{OO}}\left(r_{1} ; \eta, T\right)}{g_{\mathrm{OO}}\left(r^{\ddagger} ; \eta, T\right)} \frac{g_{\mathrm{OO}}\left(r_{2} ; \eta, T\right)}{g_{\mathrm{OO}}\left(r^{\ddagger} ; \eta, T\right)}\right],
\end{aligned}
$$

where $r_{1}$ and $r_{2}$ are the positions of the first and second shells in the rdf, $r^{\ddagger}$ is the OO distance at the jump transition state, ${ }^{13}$ $k_{B}$ is the Boltzmann constant, and $a$ is a minor, $\eta$-independent free energy contribution. As previously noted, ${ }^{22}$ the microscopic $\Delta G^{\ddagger}$ is distinct from the phenomenological $E_{a}$.

The fluctuating local structures experienced by water molecules lead to $\Delta G^{\ddagger}$ values differing by as much as $4 \mathrm{kcal} / \mathrm{mol},{ }^{13}$ thus strongly affecting the jump rate constant, especially at low temperature (Fig. 2(b)). The change in $\Delta G^{\ddagger}$ with $\eta$ is found to be approximately linear and temperature independent. ${ }^{13}$ The jump rate constant for a given local structure and a given temperature can thus be expressed within a Transition State Theory description as

$$
\begin{aligned}
k_{\text {jump }}(\eta, T) & =\kappa \frac{k_{B} T}{h} \exp \left(-\Delta G^{\ddagger} / k_{B} T\right) \\
& \simeq f(T) \exp \left(-\alpha \eta / k_{B} T\right),
\end{aligned}
$$

where $\kappa$ is the transmission factor, $\alpha$ is a constant, and $h$ is Planck's constant. $f(T)$ is determined at each temperature by fitting the average jump time $\left\langle\tau_{\text {jump }}(T)\right\rangle$ calculated directly from the simulations (Eq. (1)) with our model based on the $\eta(t)$ time fluctuations (Eq. (3)),

$$
\left\langle\tau_{\text {jump }}(T)\right\rangle=\int_{0}^{\infty} d t^{\prime}\left\langle\exp \left[-\int_{0}^{t^{\prime}} k_{\text {jump }}(\eta(t), T) d t\right]\right\rangle .
$$

Remarkably, the temperature dependence of the resulting $k_{\text {jump }}(\eta, T)$ is found to be perfectly Arrhenius,

$$
k_{\text {jump }}(\eta, T)=k_{0} \exp \left(-\frac{\alpha \eta+\Delta G_{0}^{\ddagger}}{k_{B} T}\right),
$$

with $k_{0} \simeq 0.053 \mathrm{fs}^{-1}, \alpha \simeq 5.8 \mathrm{kcal} / \mathrm{mol}$, and $\Delta G_{0}^{\ddagger}$ $\simeq-6.3 \mathrm{kcal} / \mathrm{mol} .{ }^{26}$ For a fixed local structure, there is thus nothing anomalous in the HB dynamics temperature behavior. The jump dynamics is determined by a free energy barrier which exhibits stochastic fluctuations due to the structural fluctuations.

The time-dependent HB survival probabilities calculated from the simulations (Eq. (1)) and from the model based on the $\eta$ fluctuations (Eqs. (4) and (5)) are in excellent agreement over the 235-350 K range (Fig. 3(a)).

Our model thus shows that while the jump rate constant for a given local structure (Eq. (5)) is strictly Arrhenius, the jump time averaged over the structural fluctuations (Eq. (4)) captures the non-Arrhenius behavior observed in the simulations (Fig. 3(b)). We can now use this model to determine the 

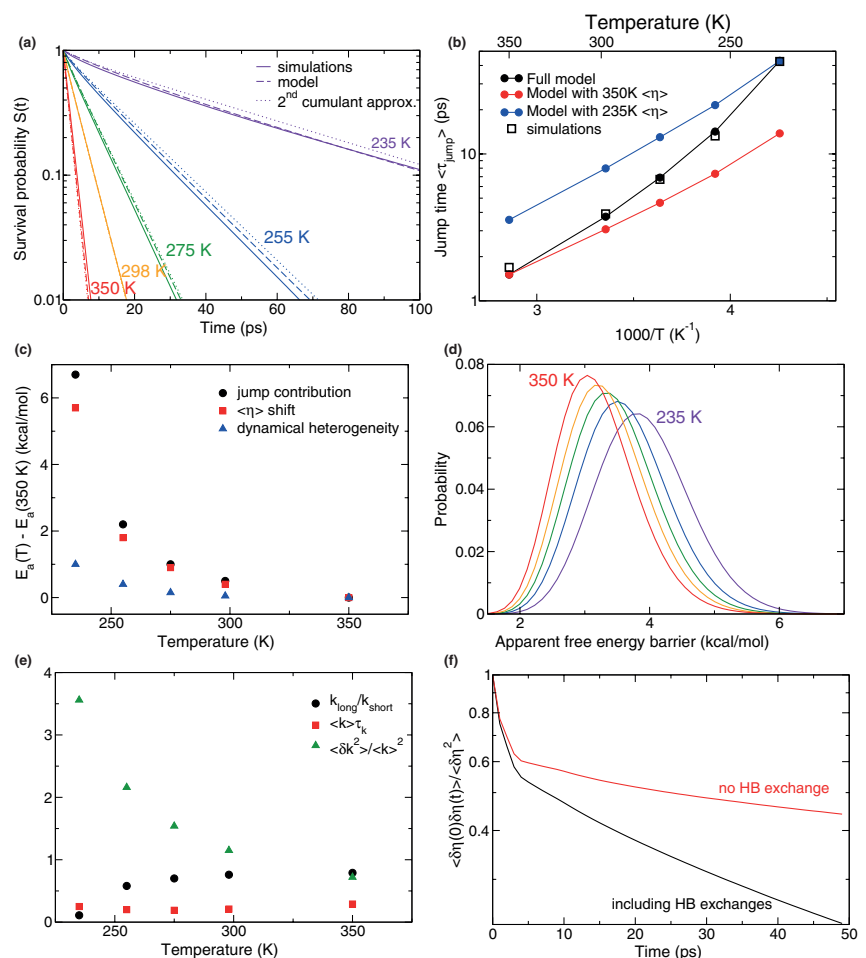

FIG. 3. (a) HB survival probability from simulations, from our model (Eq. (4)) and from its 2nd cumulant approximation (Eq. (6)); (b) Arrhenius plot of $\left\langle\tau_{\text {jump }}\right\rangle$ from simulations (Eq. (1)), from the model (Eq. (4)) and after compensating for the $\eta$ distribution shift; (c) HB jump contribution to the increase in the reorientation $E_{a}$ upon cooling from $350 \mathrm{~K}$ and its components due to the $\langle\eta\rangle$ structural shift and to the dynamical heterogeneities; (d) distribution of apparent free energy barriers $\alpha \eta+\Delta G_{0}^{\ddagger}$ (Eq. (5)) at different temperatures; (e) temperature dependence of the contributions to $k_{\text {long }} / k_{\text {short }}$; and (f) tef of $\delta \eta(t)=\eta(t)-\langle\eta\rangle$ with and without HB jump exchanges at $255 \mathrm{~K}$.

origin of this anomalous behavior and we will underline the critical importance of two features of the structural distribution, its average and its width.

First, to assess the consequences of the shift toward more tetrahedral (higher $\eta$ ) structures upon cooling (Fig. 2(b)), we recalculate $\left\langle\tau_{\text {jump }}\right\rangle$ at each temperature with Eq. (4) after shifting the $\eta$ time fluctuations by an offset which ensures that the average $\eta$ value remains constant with temperature. ${ }^{13}$ As illustrated in Fig. 3(b) for average structures constrained to be those at high $(350 \mathrm{~K})$ and low $(235 \mathrm{~K})$ temperatures respectively, removing the progressive ordering upon cooling strongly reduces the anomalous temperature dependence (the jump contribution to the reorientation $E_{a}$ change between $350 \mathrm{~K}$ and $235 \mathrm{~K}$ is reduced from $+6.6 \mathrm{kcal} / \mathrm{mol}$ to $+1.0 \mathrm{kcal} / \mathrm{mol}$, see Fig. 3(c)) and a quasi-Arrhenius behavior is recovered, i.e., $\ln \left(\left\langle\tau_{\text {jump }}\right\rangle\right)$ is almost perfectly linear with $1 / T$.

The continuous shift of the homogeneous $\eta$ distribution toward more ordered structures leads to a small increase in the apparent free energy barrier for HB exchanges (Fig. 3(d)) and to a large decrease in the jump rate constants, ${ }^{13}$ thus causing a non-Arrhenius behavior (this decrease is much reduced when the structural shift is suppressed ${ }^{13}$ ). Changes in the local structure upon cooling were recently invoked to explain some other water anomalies. ${ }^{27}$ However, a two-state descrip- tion of the water structure was employed, while its validity was challenged ${ }^{28}$ and it is not supported by our present results. ${ }^{13}$ Our results critically differ in two key aspects: a molecular quantitative connection between structure and dynamics is provided and the non-Arrhenius behavior is shown to arise from a continuous shift of a unimodal structural distribution, and not from an equilibrium between two distinct structures.

In addition to the change in the average jump rate constant with temperature, ${ }^{13}$ cooling also leads to a dramatic increase in the width of the $k_{\text {jump }}$ distribution. This increasing dynamical heterogeneity upon cooling explains both the residual (very minor) contribution to the non-Arrhenius behavior and the increasingly non-exponential orientational relaxation at low temperature (Fig. 1(b)) (it also leads to a nonexponential distribution of jump times ${ }^{13}$ ). While the width of the $\eta$ distribution and thus that of the apparent free energy barrier distribution are almost temperature independent ${ }^{13}$ (Fig. 2(b)), the decrease in thermal energy $k_{B} T$ upon cooling leads to a large increase in the range of $k_{\text {jump }}$ values. ${ }^{13}$ The impact of a broad distribution of $k_{\text {jump }}$ rate constants can be qualitatively understood by a second-order cumulant expansion of the survival probability $S(t)$ (Eq. (4)), ${ }^{29}$

$$
S(t) \simeq \exp \left[-\langle k\rangle t+\int_{0}^{t}(t-s) C(s) d s\right],
$$

where $\langle k\rangle$ is the (static) average of $k_{\text {jump }}$ over the $\eta$ distribution and $C(t)$ is the tcf of the $k_{\text {jump }}(t)$ fluctuations around the average value. At short delays, the $S(t)$ relaxation rate is $k_{\text {short }}=\langle k\rangle$, while at long delays this rate becomes $k_{\text {long }}$ $=\langle k\rangle\left(1-\frac{\left\langle\delta k^{2}\right\rangle}{\langle k\rangle} \tau_{k}\right)$, where $\left\langle\delta k^{2}\right\rangle=C(0)$ and $\tau_{k}=\int_{0}^{\infty} C(t) d t$.

This qualitative picture first explains why the relaxation of $S(t)$ at low temperature resembles a bi-exponential decay (in contrast to the commonly assumed stretched-exponential decay) (Fig. 1(b)). While at high temperature the two relaxation rates are approximately equal (leading to a single exponential decay of $S(t)$ ), upon cooling $k_{\text {long }}$ becomes markedly slower than $k_{\text {short }}$, due to the increase in the $\left\langle\delta k^{2}\right\rangle \tau_{k} /\langle k\rangle$ term, which can be decomposed as a product of two factors $\left(\tau_{k}\langle k\rangle\right) \times\left(\left\langle\delta k^{2}\right\rangle /\langle k\rangle^{2}\right)$, which we now analyze.

This growing discrepancy is not due to a freezing of rate fluctuations upon cooling, since $\tau_{k}\langle k\rangle$, which approximately represents the ratio between the respective timescales of structural relaxation $\left(\tau_{k}\right)$ and of jumps $(1 /\langle k\rangle)$, is almost temperature independent (Fig. 3(e)). HB jumps and $\eta$ fluctuations are connected, since water molecules which remain hydrogenbonded to their four closest neighbors do not experience a full $\eta$ relaxation (Fig. 3(f)). Since a jump in one of the four HB formed on average by a water molecule can randomize the water $\eta$ value, molecular structural relaxation is approximately four times faster than HB jumps, which may explain why $\tau_{k}\langle k\rangle \simeq 1 / 4$ (Fig. 3(e)).

The growing dynamical disorder upon cooling is instead due to the broadening range of $k_{\text {jump }}$ values, ${ }^{13}$ leading to the dramatic increase in $\left\langle\delta k^{2}\right\rangle /\langle k\rangle^{2}$ (Fig. 3(e)) and thus explains why $k_{\text {long }}$ increasingly differs from $k_{\text {short }}$.

Interestingly, our model can provide an explanation to the origin of the dynamical heterogeneities recently observed in 
liquid water at room temperature: ${ }^{30}$ the experimental $500 \mathrm{fs}$ timescale measured for the relaxation of these heterogeneities is consistent with the $\tau_{k}$ relaxation time measured to be $\simeq 600$ fs at $298 \mathrm{~K}$ in our simulations. These heterogeneities simply arise from structural fluctuations within a single Gaussian distribution which lead to fluctuations in the HB dynamics, but they do not imply the existence of distinct interconverting structures.

Our simulations and our model thus show that the increase in the apparent $E_{a}$ of water reorientation between $350 \mathrm{~K}$ and $235 \mathrm{~K}$ (ca. $+8 \mathrm{kcal} / \mathrm{mol}$ ) is mainly due to the continuous progressive shift toward more ordered structures upon cooling (for 65\%), with additional contributions coming from the increase in viscosity $(20 \%)$ and from the growing dynamical disorder (15\%). The HB dynamics exhibits a regular Arrhenius behavior for a fixed local structure. No change in the reorientation mechanism occurs at low temperature. While an equilibrium between distinct structures cannot be excluded, it is not found in our simulations and we show that the non-Arrhenius behavior can be explained with a homogeneous structure distribution. The anomalous temperature dependence does not imply either an increasingly collective character of some motions leading to a dynamical arrest and a glass transition upon cooling. ${ }^{10}$ Although the shift toward more ordered structures results in an increasing $E_{a}$, it does not lead to any divergence of water dynamics, in contrast with the popular mode-coupling approach. The absence of such a critical temperature where dynamics diverges is consistent with recent results showing the structural instability of liquid water below the homogeneous nucleation temperature, ${ }^{31}$ which indicates it is not trapped in a glassy state, as already suggested for this water model. ${ }^{6,12}$ At very low temperature, a transition to a regime where $E_{a}$ becomes constant, i.e., a fragile to strong transition, ${ }^{32}$ may arise within our model if the structural distribution stops shifting. The importance of the shift to more tetrahedrally ordered structures for water dynamics also echoes the recent suggestion that the increase in four-coordinated waters explains the water anomalous thermodynamics. ${ }^{31}$

An extension of the present model is underway to describe structural fluctuations in the hydration shell of hydrophobic solutes and proteins and explain the unexpected temperature dependence of the water retardation factor in the shell relative to the bulk. ${ }^{33}$
We thank F. Sterpone and E. Duboué-Dijon for valuable suggestions and J. T. Hynes, W. H. Thompson, P. Debenedetti, and M. D. Fayer for their critical reading of the paper.

${ }^{1}$ K. Ito, C. T. Moynihan, and C. A. Angell, Nature (London) 398, 492 (1999).

${ }^{2}$ P. G. Debenedetti, J. Phys. Condens. Matter 15, R1669 (2003).

${ }^{3}$ E. Lang and H. D. Lüdemann, J. Chem. Phys. 67, 718 (1999).

${ }^{4}$ J. Teixeira, M. C. Bellissent-Funel, S. H. Chen, and A. J. Dianoux, Phys. Rev. A 31, 1913 (1985).

${ }^{5}$ J. Qvist, H. Schober, and B. Halle, J. Chem. Phys. 134, 144508 (2011).

${ }^{6}$ D. Rozmanov and P. G. Kusalik, J. Chem. Phys. 136, 044507 (2012).

${ }^{7}$ L. Xu, P. Kumar, S. V. Buldyrev, S.-H. H. Chen, P. H. Poole, F. Sciortino, and H. E. Stanley, Proc. Natl. Acad. Sci. U.S.A. 102, 16558 (2005).

${ }^{8}$ C. Huang et al., Proc. Natl. Acad. Sci. U.S.A. 106, 15214 (2009).

${ }^{9}$ R. A. Nicodemus, S. A. Corcelli, J. L. Skinner, and A. Tokmakoff, J. Phys. Chem. B 115, 5604 (2011).

${ }^{10}$ P. Gallo, F. Sciortino, P. Tartaglia, and S. H. Chen, Phys. Rev. Lett. 76, 2730 (1996).

${ }^{11}$ J. Swenson and J. Teixeira, J. Chem. Phys. 132, 014508 (2010).

${ }^{12}$ J. L. F. Abascal and C. Vega, J. Chem. Phys. 133, 234502 (2010).

${ }^{13}$ See supplementary material at http://dx.doi.org/10.1063/1.4737390 for methodology, jump times and Voronoi asphericity.

${ }^{14}$ K. J. Tielrooij, C. Petersen, Y. L. A. Rezus, and H. J. Bakker, Chem. Phys. Lett. 471, 71 (2009).

${ }^{15}$ R. Richert, J. Phys. Condens. Matter 14, R703 (2002).

${ }^{16}$ M. Mazza, N. Giovambattista, F. Starr, and H. Stanley, Phys. Rev. Lett. 96, 057803 (2006).

${ }^{17}$ B. Jana, R. S. Singh, and B. Bagchi, Phys. Chem. Chem. Phys. 13, 16220 (2011).

${ }^{18}$ R. Ludwig, F. Weinhold, and T. C. Farrar, J. Chem. Phys. 103, 6941 (1995).

${ }^{19}$ D. Laage and J. T. Hynes, Science 311, 832 (2006).

${ }^{20}$ D. Laage, G. Stirnemann, F. Sterpone, R. Rey, and J. T. Hynes, Annu. Rev. Phys. Chem. 62, 395 (2011).

${ }^{21}$ M. Mazza, N. Giovambattista, H. Stanley, and F. Starr, Phys. Rev. E 76, 031203 (2007)

${ }^{22}$ D. Laage and J. T. Hynes, J. Phys. Chem. B 112, 14230 (2008).

${ }^{23}$ J. R. Errington and P. G. Debenedetti, Nature (London) 409, 318 (2001).

${ }^{24}$ G. Ruocco, M. Sampoli, and R. Vallauri, J. Chem. Phys. 96, 6167 (1992).

${ }^{25}$ Y. L. Yeh and C. Y. Mou, J. Phys. Chem. B 103, 3699 (1999).

${ }^{26}$ In this effective description, the temperature dependence of $\kappa$ (Ref. 22) is included in $\Delta G_{0}^{\ddagger}$. While $\Delta G_{0}^{\ddagger}<0, \alpha \eta+\Delta G_{0}^{\ddagger}$ is always positive for the $\eta$-range of interest.

${ }^{27}$ L. Xu, F. Mallamace, Z. Yan, F. W. Starr, S. V. Buldyrev, and H. E. Stanley, Nat. Phys. 5, 565 (2009).

${ }^{28}$ G. N. I. Clark, G. L. Hura, J. Teixeira, A. K. Soper, and T. Head-Gordon, Proc. Natl. Acad. Sci. U.S.A. 107, 14003 (2010).

${ }^{29}$ While a second-order truncation is not exact for the jump rate constants which are not Gaussian, Fig. 3(a) shows that this approximation is numerically acceptable over the studied temperature range.

${ }^{30}$ S. Garrett-Roe, F. Perakis, F. Rao, and P. Hamm, J. Phys. Chem. B 115, 6976 (2011).

${ }^{31}$ E. B. Moore and V. Molinero, Nature (London) 479, 506 (2011).

${ }^{32}$ C. A. Angell, Science 319, 582 (2008).

${ }^{33}$ C. Mattea, J. Qvist, and B. Halle, Biophys. J. 95, 2951 (2008). 